\title{
Recursos hídricos y desarrollo sostenible: requisitos para la planificación y gestión compartida entre España y Portugal
}

\author{
Alejandro LÓPEZ LÓPEZ \\ Departamento Ecología Humana y Medio Ambiente \\ Universidad Complutense de Madrid \\ lopezal@pdi.ucm.es
}

Recibido: 11 de Abril de 2001

Enviado a evaluar: 12 de Abril de 2011

Aceptado: 5 de Octubre de 2011

\section{RESUMEN}

La tierra tiene una cantidad de agua constante pero sufre de estrés hídrico y los pronósticos para el futuro no son optimistas. De ahí que la ONU en los "Objetivos del Desarrollo del Milenio para el 2015" haga espacialísima referencia al tema del agua. El artículo enfatiza los indicadores de sostenibilidad para los recursos hídricos y propone un modelo económico de eficiencia eco-social para la planificación y gestión compartida entre España y Portugal.

Palabras clave: Recursos Hídricos. Desarrollo Sostenible. Planificación integrada en agua y energía. Planificación y gestión compartida entre España y Portugal en materia de agua

\section{Water resources and sustainable development: planning requirements and shared management between Spain and Portugal}

\begin{abstract}
The Earth has a constant quantity of water, but suffers hydric stress and forecast of future is not optimistic. Thus, the UN in the "Millennium Devolopment Goals for 2015" establishes special reference to the issues of water. This paper highlights the indicators of sustainability for the hydirc resources and proposes an ecosistemic model of eco-social efficiency for the sharing plannig and management between Spain and Portugal.
\end{abstract}

Keywords: Hydric resources; Sustainable development; Integrated planning for water and energy; Sharing planning and management between and Portual for water issues. 
Ressouces hydriques et développement durable: conditions pour la planification et la géstion partagée entre l'Espagne et le Portugal

\section{RESUMÉ}

La Terre a une quantité constante d'eau, mais souffre de stress hydrique et la prévision de l'avenir n'est pas optimiste. Ainsi, les Nations Unies dans les “Objectifs du Millénaire pour le développement en 2015", établit une réference particulère à la question d'eau. Ce document met en évidence les indicateurs de durabilité pour les ressources hydriques et propose un modèle éco-systémique de l'efficacité éco-sociale de la planification et la gestion de partage entre l'Espagne et le Portugal

Mots clé: Ressources hydriques, Développement durable, Planification intégrée de l'eau et l'énergie, Planification et la gestion de partage entre l'Espagne et le Portugal pour les questions de l'eau.

\section{PRESENTACIÓN 1}

La Tierra tiene una cantidad de agua constante pero sufre de estrés hídrico, y los pronósticos para el futuro no son optimistas. La ONU calcula que para el año 2025 unos 1.800 millones de personas vivirán en regiones afectadas por una escasez absoluta de agua que se deberá, entre otras causas, al crecimiento demográfico, la contaminación y las manipulaciones del territorio (Falkenmark). Esta monografía de VANGUARDIA DOSSIER analiza las limitaciones políticas, institucionales y económicas de una crisis que desde hace tiempo es una realidad para millones de personas, ya que los recursos están repartidos desigualmente, y tiene más que ver con la mala gestión de los recursos que con la escasez (Rijsberman y Manning).

Uno de los mayores desafíos del siglo XXI es asegurar la suficiente energía y agua para el bienestar de la humanidad, manteniendo, al mismo tiempo, la salud económica, integridad y capacidad de recuperación de las cuencas hidrográficas (Totten y Zurita). ¿Qué significa, entonces, el estrés hídrico? Significa que el índice de agua disponible per cápita cae por debajo del nivel capaz de satisfacer las necesidades de un país. La ONU considera que el agua constituye un derecho humano básico, pero para muchos este derecho sigue siendo teórico (Conca).

La crisis del agua es una crisis de gobernabilidad (Fernández-Jáuregui) El modelo de posguerra fue la construcción de presas. La situación ha cambiado. Una opción

1 Este artículo forma parte del Proyecto de Investigación : "Planificación estratégica y gestión compartida de los recursos hídricos hispano - lusos". Plan Nacional I+D. Convocatoria 2010 de Investigación Fundamental no orientada. Dirigido por el Prof. Dr. D. Julián Mora Aliseda -(Universidad de Extremadura, Campus de Cáceres y Universidad de Lisboa)

Fue igualmente materia de Ponencia con el mismo título en el V Congreso Internacional de “Ordenación del Territorio y Recursos Hídricos Internacionales, Lisboa, 27 y 28 de octubre 2010. 
es la desalinización; otra es el reciclaje del agua, y una tercera es la reducción de la demanda y la redistribución (Skinner). En otras palabras, ha llegado la hora de las estrategias de la demanda - ahorro y mejora del uso- y de la conservación de los ecosistemas acuáticos, cuya gestión como recursos críticos que son ya es urgente, antes de que la situación sea irreversible (Revenga).

El agua hay que gestionarla racionalmente. Pero la buena gestión es un proceso constante de conflictos. Las relaciones entre el agua y la salud humana, por ejemplo, son múltiple y complejas (Neira y Bos). Y abundan las tensiones políticas. Kofi Annan, anterior secretario general de la ONU, ha advertido que "la competencia feroz por el agua dulce puede convertirse en una fuente de conflictos y guerra en el futuro". Para algunos expertos esta afirmación es un hidromito. En el último medio siglo, sólo se han registrado 37 disputas violentas por el agua, mientras se han firmado 157 tratados. El agua, en cualquier caso, cada vez es más tratada como un tema de seguridad (Wolf). En el mundo existen 263 cuencas hídricas entre dos o más países.

El agua no tiene precio, pero hay que pagarla. Actualmente existe una gran presión para privatizar y mercantilizar el agua. Para unos, la participación del sector privado puede ser una herramienta útil (Prager). Otros, por el contrario, consideran que lo último que se puede esperar del mercado es que se ocupe de valores no mercantiles (Postel). Es difícil, sin embargo, que el agua escape a las leyes básicas de la economía. Todos los bienes económicos son, por definición, escasos, y de lo que se trata es de gestionar la escasez desde la racionalidad (Arrojo). El desafío planteado es si las instituciones y organismos evolucionarán con la suficiente rapidez para posibilitar la cooperación ante las demandas, la rivalidad y las tensiones que provoca el estrés hídrico. (BATALLA, Xavier: VANGUARDIA Dossier nº 21: "AGUA: el desafío del siglo XXI" octubre/noviembre, 2006)

\section{INTRODUCCIÓN}

El V Programa de acción en materia de medio ambiente de la Comunidad Europea (1992) además de plantear abiertamente la nueva frontera del Desarrollo Sostenible orientado al Medio ambiente, sigue la trayectoria de los Programas I, II, III y IV en materia de Medio Ambiente de la entonces CEE, así como el conjunto de Directivas sobre el tema del agua en los distintos usos por parte de los ciudadanos para salvaguardar la salud de su población y atender a las necesidades agrícolas, industriales y del turismo (especialmente las aguas de baño en el litoral prevalentemente en el Mediterráneo).

Así pues entre las cuestiones prioritarias e importantes a escala comunitaria y en el ámbito internacional, la Comunidad consolida su compromiso en todas las iniciativas destinadas a proteger los mares regionales y los ríos internacionales. Además de su participación en los acuerdos vigentes para la protección del Mediterráneo y del mar del Norte, la Comunidad tiene intención de convertirse en parte contratante del Convenio de Helsinki sobre la protección del medio ambiente marino en la Región del mar Báltico. Participa también en las negociaciones para la elaboración de un Convenio marco sobre la protección y el uso de los ríos transfronterizos y los 
lagos internacionales, y en una serie de acuerdos sobre varias cuencas fluviales europeas, entre las que figura la del Danubio. La Comunidad Europea ya suscribió en 1990, junto con Alemania y la República Checoslovaca, un Convenio sobre el Elba, y próximamente se celebrará otro sobre el Oder. A través del programa MEDSPA, la Comunidad apoya activamente el Programa de Medio Ambiente para el Mediterráneo lanzado conjuntamente por el Banco Mundial y el BEI. Este plan proporciona un buen ejemplo de cooperación interinstitucional para la protección del medio ambiente que podría ampliarse a otras regiones.

Sin embargo, como ha puesto de manifiesto el VI Programa en materia de Medio Ambiente (2001-2010) "Medio ambiente 2010: el futuro está en nuestras manos" a pesar de las mejoras observadas en algunos campos, no obstante, seguimos enfrentados a una serie de problemas persistentes. Especialmente preocupantes son el cambio climático, la merma de biodiversidad y habitats naturales, la pérdida y degradación de suelos, el creciente volumen de residuos, la acumulación de sustancias químicas en el Medio Ambiente, el ruido y algunos contaminantes del agua y del aire.

El problema de los recursos hídricos consiste en que reconociendo que las dos o tres últimas décadas se han registrado mejoras significativas en numerosos aspectos de la calidad del agua, pero los datos y las previsiones actuales indican que todavía nos enfrentamos a determinados problemas y tendencias negativas, por ejemplo, en lo que se refiere a la contaminación de las aguas subterráneas por los plaguicidas y los nitratos a causa de las actividades agrícolas, y a pesar de que la calidad de las aguas de baño en las costas haya mejorado gradualmente, todavía queda camino por recorrer en muchos sitios. Más aún, algunas regiones corren el riesgo de experimentar tendencias insostenibles, especialmente en la Europa meriodional.

\section{DESARROLLO SOSTENIBLE}

En la publicación de la Unión Internacional para la Conservación de la Naturaleza (UICN) de 1980 aparece por primera vez el concepto de "Desarrollo compatible". Este concepto vendrá precisándose en los años sucesivos con la introducción del concepto de "Ecodesarrollo" por parte de Ignacy SACHS (1981), se trata de un "desarrollo socialmente deseable, económicamente viable y ecológicamente prudente y respetuoso con la naturaleza"

Como dijo más tarde la señora BRUNDTLAND en la presentación de Nuestro Futuro común, (Oslo, 20 de marzo de 1987) y cuyo informe de la Convención de Medio Ambiente y del Desarrollo (ONU) vio su publicación en castellano en Alianza Universidad, Madrid, 1988,: "Lo que actualmente se necesita es una nueva era de crecimiento económico, un crecimiento que sea poderoso a la vez que sostenible social y medioambientalmente".

\subsection{LA INTERCONEXIÓN DE LA CRISIS}

Los cambios conexos han entrelazado de varias maneras la economía y la ecología mundiales. En el pasado nos preocupábamos de los efectos del crecimiento eco- 
nómico sobre el medio ambiente. Ahora nos vemos obligados a preocuparnos de la presión ecológica -el deterioro de los suelos, las aguas, la atmósfera y los bosquessobre nuestras perspectivas económicas.

En el pasado más reciente nos hemos visto obligados a hacer frente a un notable aumento de la interdependencia económica de las naciones. Y ahora estamos obligados a acostumbrarnos a una creciente interdependencia ecológica entre esas mismas naciones. La ecología y la economía se entreveran cada vez más - en los planos local, regional, nacional y mundial- hasta formas una red de causa y efectos.

\subsection{EL DESARROLLO SOSTENIBLE}

Asegurar que satisfaga las necesidades del presente sin comprometer la capacidad de las futuras generaciones, para satisfacer las propias. El concepto de desarrollo sostenible implica límites - no límites absolutos, sino limitaciones que imponen a los recursos del medio ambiente el estado actual de la tecnología y de la organización social y la capacidad de las biosfera de absorber los efectos de las actividades humanas-, pero tanto la tecnología como la organización social pueden ser ordenadas y mejoradas de manera que abran el camino a una nueva era de crecimiento económico.

Pero el desarrollo sostenible no es un estado de armonía fijo, sino un proceso de cambio por el que la explotación de los recursos, la dirección de las inversiones, la orientación de las progresos tecnológicos y la modificación de las instituciones concurran en las necesidades tanto presentes como futuras.

No se puede encontrar una fórmula única de desarrollo sostenible, debido a que los sistemas económicos y sociales y las condiciones ecológicas difieren enormemente de una país a otro. Cada país tendrá que resolver sus propias implicaciones políticas. Pero cualquiera que sean las diferencias, el desarrollo sostenible deberá considerarse como un objetivo global.

Es sabido que cinco años más tarde tuvo lugar la Conferencia de Río de Janeiro (1992) o también conocida como la Cumbre de la Tierra donde se tipificó definitivamente la filosofía, el concepto y el programa para la acción del "Desarrollo Sostenible". En dicha ocasión, ha visto la luz sobre la base de la negociación la Agenda 21, o sea, un programa de acción para el siglo XXI con el objetivo del Desarrollo Sostenible para encauzar conjuntamente los esfuerzos de las diferentes naciones y para conjugar o compatibilizar el desarrollo con la calidad del Medio Ambiente.

En dicho planteamiento se reconoce la creciente disparidad de renta entre ricos y pobres, la deuda creciente de los países del Tercer Mundo y la diferencia de acceso a los recursos naturales entre el Norte y el Sur del Ecosistema Mundial.

La fase de desarrollo sostenible se ha impuesto con los años como la necesidad de afrontar el problema de Medio Ambiente, de la economía y de la sociedad en sus variadas facetas de modo global y con el apoyo de las leyes nacionales y regionales encaminadas hacia dicho fin.

Este modo de afrontar el problema ha madurado tras diversas experiencias ya que en los años 70 se veían a los países industrialmente más avanzados proyectados hacia 
una explotación sin límites de los recursos naturales con el riesgo de poner en dificultad el ecosistema y de acentuar todavía más la distancia entre el Norte y el Sur del Planeta.

La culminación de la filosofía y la propuesta definitiva de desarrollo sostenible tuvo lugar en la Conferencia de Río 92, de la cual en este texto me voy a limitar a enumerar algunos de los principales principios de la Declaración de Río sobre el desarrollo sostenible:

1 Los seres humanos son el centro del desarrollo sostenible. El ser humano tiene derecho a una vida productiva y saludable en armonía con la naturaleza.

2 Los Estados conforme a la carta de las Naciones Unidas y a la legislación internacional tienen el derecho soberano de exportar sus propios recursos de acuerdo con sus políticas ambientales y de desarrollo.

3 Debe satisfacer el derecho al desarrollo así como hacer frente de una forma equitativa a las necesidades de las generaciones presentes y futuras.

4 La protección del medio ambiente debe ser parte integrante del proceso de desarrollo para lograr el desarrollo sostenible.

5 Todos los Estados y todas las personas deberían colaborar en la tarea fundamental de erradicar la pobreza como un elemento indispensable del desarrollo sostenible.

6 Los Estados deberían reducir o eliminar los modelos insostenibles de producción y consumo.

7 Los Estados deberían cooperar para fortalecer su capacidad endógena para construir mejor su desarrollo sostenible.

8 La mejor forma para tratar los problemas ambientales consiste en la participación de todos los ciudadanos implicados.

9 Debería utilizarse la Evaluación de Impacto Ambiental para controlar las actividades.

10 La población indígena y las comunidades locales tiene un papel importante en la gestión ambiental debido a sus conocimientos y prácticas tradicionales.

\section{INDICADORES DE SOSTENIBILIDAD DE LOS RECURSOS HÍDRICOS}

\subsection{LA DIRECTIVA EUROPEA DEL AGUA}

Lo más destacable es la adopción de una nueva Directiva Marco de Aguas. Con ella se extiende la protección de la capa freática a todas las aguas y se obliga a utilizar un sistema de precios de uso del agua, para favorecer su conservación.

Pero en la Directiva no sólo se habla de la calidad del agua sino también de su gestión. Se pone énfasis en el principio de responsabilidad compartida de los agentes implicados a diferentes niveles, tanto para la calidad del recurso como para su gestión. En este sentido, se insta a las diferentes escalas de gobierno a que fomenten 
un consumo eficiente tanto a nivel doméstico como en las actividades económicas, particularmente en la agricultura.

Los tres pilares básicos en los que se sustenta la Directiva son la ecología, la economía y la participación pública. Estos elementos deben interrelacionarse, para lograr un objetivo común, la gestión sostenible. El motivo por el cual hay que relacionarlos es el hecho de que la protección del medioambiente es esencial para la calidad de vida de las generaciones actuales y futuras. La relación con la economía viene dada por el estímulo para la innovación y el negocio que debe generarse para lograr un crecimiento continuo sostenible. Por si fuera poco, para ello debe tenerse en cuenta además la participación pública activa.

En consonancia con todos estos criterios se encuentra la denominada "Nueva Cultura del Agua". La novedad principal que se establece es que el medio ambiente se convierte en un objetivo fundamental de la gestión hídrica.

Las acciones que deben llevarse a cabo en esta línea son la de la aplicación de la Directiva en esos tres ámbitos (económico, ecológico y social); y también la de integrarla en las demás políticas relativas a la calidad del agua, en la PAC y en la Política de Desarrollo Regional: entre otras acciones.

\subsection{LOS BENEFICIOS DE LA PLANIFICACIÓN INTEGRADA EN AGUA Y ENERGÍA.}

Uno de los mayores desafíos del siglo XXI consiste en asegurar la provisión de suficiente energía y agua para el bienestar de la población, al mismo tiempo que se preserva la salud ecológica, la integridad y la capacidad de recuperación de las cuencas hidrográficas, desde las cumbre hasta los océanos.

El cambio climático que el planeta está experimentando presenta múltiples amenazas, por lo que un componente clave para enfrentar este desafío es diseñar y aplicar políticas efectivas y prácticas innovadoras que permitan una gestión que se adapte a los cambios ecohidrológicos inducidos por las modificaciones climáticas.

El agua dulce es ahora el precioso e indispensable cimiento de una vida sostenible y de un bienestar próspero y es el torrente sanguíneo y el hogar de la más rica biodiversidad y de las especies más antiguas que habitan el planeta. Los ecosistemas de agua dulce contienen concentraciones de especies únicas que presentan una mayor proporción de diversidad en relación a su área, muy superior a la de los ecosistemas terrestres y marinos. Por citar un ejemplo, los sistemas de agua dulce contienen cerca de la mitad de las especies de peces globales, una concentración 4.000 veces mayor que la de los océanos. Por otro lado, las extinciones de especies documentadas en agua dulce son diez veces superiores que las de los océanos.

El Proceso Integrado de Planificación de Agua y Energía (PIPAE) es el modelo para la provisión de servicios de energía y agua al menor coste y riesgo que se ha aplicado en California. Se trata de una combinación de innovaciones políticas y de regulaciones que se aplicaron con éxito al sistema de provisión eléctrica hace varias décadas - por ejemplo, incentivos para los sistemas de provisión de servicios y estándares para los electrodomésticos y construcciones estatales-. Este acuerdo cobra 
mayor importancia por cuanto mientras se registraba un fuerte crecimento económico, el consumo de electricidad permaneció sin variaciones como resultado de las mejoras continuas a la eficiencia de electrodomésticos, bombillas, equipos de oficina, motores, edificios y fábricas de manufactura. Mientras en el resto de Estados Unidos se experimentaba un crecimiento anual en el consumo eléctrico del 2 por ciento, en California se frenó el 50 por ciento de la expansión de su sistema eléctrico. Hasta 2004, las facturas de electricidad de los consumidores de California registraron una reducción total de 16.000 millones de dólares o mil dólares anuales netos de ahorro por familia. Una parte de beneficio adicional en el ahorro fue debida a las reducciones sin coste alguno en las emisiones de gases de efecto invernadero.

Una encuesta a nivel estatal sobre las mejores prácticas de gestión implementadas por 86 agencias de agua, encontraron ahorros significativos de agua y energía en media docena de iniciativas - desde inodoros con flujos ultrabajos, paisajismo de conservación, reembolsos por compras de lavadoras de alta eficiencia, medición más exacta con tazas de bienes de mercado, modificaciones en plomería-. Se comprobó que se habían ahorrado anualmente más de cien millones de metros cúbicos (100.000 millones de litros) de agua y 254 millones de $\mathrm{kWhs}$ de electricidad. A lo largo de la aplicación de estas medidas, el valor presente neto de esta energía y de agua ahorradas excedieron los 200 millones de dólares. El costo de la aplicación de estas medidas de eficiencia de agua alcanzó un promedio de 0,31 dólares por $\mathrm{m}^{3}$, en un rando de 0,04 a 0,58 .

E1 Instituto Pacífico evaluó el nivel de oportunidades de agua disponibles hasta 2030 y concluyó que California puede reducir un 20 por ciento el consumo de agua, una reducción equivalente a cerca de 10,5 billones de litros $\left(10,5 \mathrm{Km}^{3}\right)$ por año, a pesar del substancial crecimiento poblacional y económico. El estudio con un enfoque de abajo hacia arriba, estima el uso futuro del agua multiplicando el número de dispositivos, como inodoros, por sus requisitos técnicos de agua. Un número de recomendaciones claves fueron presentadas para asegurar que este futuro de consumo sostenible de agua se convierta en una realidad:

1 Las políticas de precios que subvencionan el uso inadecuado del agua deben ser eliminadas

2 Promover el uso de tecnologías y prácticas eficientes para el agua a través de estándares más estrictos de efíciencia, reembolsos extensivos por instalar electrodomésticos de menor consumo, mejores prácticas de gestión obligatorias y que se hagan cumplir, y desarrollo y despliegue de técnicas de alta eficiencia para irrigar y nuevos tipos de cultivos.

3 Expandir herramientas educativas como notas informativas en los electrodomésticos, redes de información por internet para agricultores sobre el clima, condiciones del suelo y necesidades de agua de los cultivos.

4 Mejorar la planificación combinada de agua y tierra para asegurar la protección de cuencas hídricas.

La planificación integrada de recursos permite identificar oportunidades de mejora de la calidad social y ambiental que no se contemplan en los procesos tradicionales. 
Cuando los proyectos son planificados de una manera integrada, identificando oportunidades que por barreras institucionales y políticas no habían sido salvadas antes, se pueden obtener beneficios no calculados previamente y que permiten una gestión de los recursos más eficientes y generan un significado ahorro financiero. (TOTTEN, Michael / ZURITA, Patricia VANGUARDIA ob. Cit pág: 98-107)

\subsection{LA CONFEDERACIÓN HIDROGRÁFICA DEL TAJO Y EL TRASVASE TAJO - SEGURA}

En un contexto de recursos hídricos insuficientes y de mala calidad, los regadíos y los abastecimientos urbano-turísticos del Bajo Segura, Bajo Vinalopó, Campo de Alicante, Campo de Cartagena y Valle de Guadalentín, litoral de Águilas-Mazarrón dependen de modo estratégico del agua del trasvase, que ha adquirido un incalculable valor para Murcia y Alicante al aportar unos recursos de gran calidad para los suministros agrarios y los abastecimientos de agua potable, que gestiona en alta la Mancomunidad de los Canales del Taibilla. También ha servido para paliar la sobreexplotación que padecen muchos acuíferos murcianos y alicantinos.

La ejecución de esta infraestructura, sin embargo, genera diferentes percepciones según nos encontremos en la cuenca cedente o receptora.

En estos veinticinco años de funcionamiento del acueducto Tajo-Segura se ha evidenciado lo necesario que fue su construcción para asegurar el desarrollo económico y social de Murcia y Alicante, pues sus aguas destinadas al abastecimiento urbano han sido, sin duda alguna, las impulsoras de la actividad turística litoral y de algunas industrias. Igualmente, las nuevas áreas regadas del Campo de Cartagena, Bajo Segura y valle de Guadalentín, constituyen en la actualidad la superficie de regadío (130.000 hectáreas) de mayor rendimiento económico y social de España, excepción de Almería, como lo atestigua el valor, 2.265 millones de euros, de las exportaciones agroalimentarias murcianas en el año 2002.

Se puede afirmar que hoy en día la riqueza productiva de Alicante y Murcia en un 50 $\%$ depende de la aportación de las aguas del Tajo, por lo que estos territorios receptores deben ser solidarios con los cedentes y no olvidar nunca su dependencia de aquéllos.

El elevado rendimiento que el agua en el abastecimiento urbano-turísticos-industrial y en la horticultura de ciclo manipulado ha generado y, ante su carencia, en los últimos años se ha apostado por un nuevo trasvase desde el Ebro y, en tanto se concreta y materializa, se ha recurrido a la desalación de aguas salitrosas continentales y marinas, a fin de no cercenar el desarrollo socioeconómico alcanzado. Ahora bien, se hace necesario a todas luces una adecuada ordenación territorial que evite desmanes y despilfarros en el uso del agua y compensaciones económicas.

\subsection{EL DEBATIDO Y NO NACIDO PLAN HIDROLÓGICO ESPAÑOL}

En el caso concreto de la Península Ibérica, la existencia de dos territorios biogeográficos distintos, la "Iberia seca" y la "Iberia húmeda", influye muy notablemente en el uso del líquido elemento. Pues, el carácter "seco" no es tanto consecuencia de unas precipitaciones escasas -que también en algunas zonas-, sino que éstas son irregulares, 
tormentosas y con una alta evapo-transpiración, presentando un "balance hídrico natural" deficitario a lo largo de buena parte del año, con rasgos marcadamente mediterráneos. Ëste se ha compensado a través del uso de agua subterránea, y, mediante la selección de plantas y especies animales que se adaptaban a este tipo de necesidades. Además, esto se ha visto complementado por dos hechos recientes la expansión imparable del sector turístico residencial, y, de la agricultura hortofrutícola.

Nos encontramos por tanto ante una realidad tangible, todas las cuencas hidrográficas presentan un déficit natural de agua de calidad - excepción hecha del norte y noroeste-, dependiendo de cuestiones políticas el que haya o no excedente de agua de calidad. Así, pues, cómo corregir el desequilibrio hídrico, solventando obstáculos orográficos que inciden notablemente en el reparto del agua. Además, la continua expansión de los regadíos en áreas como la Meseta está acortando aún más la posibilidad de trasvasar los excedentes. El caso concreto del trasvase Tajo-Segura nos muestra la falta de previsión, ya que no se intuyó siquiera que en la Mancha se iban a poner en regadío casi 150.000 hectáreas, por lo que amén de la explotación de las aguas subterráneas, se necesitaría también el agua trasvasada.

Por otra parte, no podemos olvidarnos que tras un siglo de construcción de grandes obras hidráulicas promovidas por el Estado, no se ha conseguido satisfacer la demanda de la población, ni superar los efectos negativos de las sucesivas sequías, aunque sí transformar y degradar las cuencas hidrográficas (tanto en el ámbito superficial como en el subterráneo). Todo ello unido a un notable aumento del coste económico y ecológico de las nuevas obras; de hecho, al propugnarse una oferta de agua a bajo precio, se potenciaron políticas de gestión y uso muy poco racionales (al transmutar la cultura tradicional que facilitaba a la población el convivir con la escasez de agua, característica del clima mediterráneo, por otra marcada por la abundancia sin límite, se ha caído en la indiferencia en el uso del agua, y por los costes de los proyectos).

A lo expuesto debemos añadir que durante los últimos años el interés por el hipotético "Cambio Climático" inducido por el efecto invernadero está suponiendo la realización de distintas investigaciones. Los aspectos más destacados en relación con este problema, desde el punto de vista socioeconómico, radica en sus posibles efectos. Según un trabajo de los profesores F.J. Ayala y A. Iglesias -(2000) "Impactos del posible cambio climático sobre los recursos hídricos, el diseño y la planificación hidrológica en la España peninsular", BBVA, El Campo, pp. 201-222- uno de los mecanismos naturales que se vería afectado, sería el Balance Hídrico, y por tanto los recursos hídricos disponibles. Se trata de un tema de especial interés en los países de clima mediterráneo como España, dónde, aparte del abastecimiento urbano, sectores importantes como el turismo y la agricultura de regadío, dependen del agua disponible. La agricultura de secano, al cambiar el agua disponible en el suelo y el régimen térmico resultaría también afectada.

Desde el punto de vista de las Cuencas, teniendo en cuenta que los resultados son valores medios, tenemos:

El posible "Cambio Climático" produciría una reducción global de los Recursos Hídricos (superficiales + subterráneos) del 17 \% para el 2060 (Horizonte de proyec- 
to medio de las grandes actuaciones hidráulicas) en la España Peninsular, junto a un aumento de la variabilidad interanual de las mismas. Estos efectos serían mayores en la mitad Sur de España.

Respecto de los Recursos Regulados, las reducciones porcentuales serían del mismo orden de las de los Recursos Hídricos para demanda continua (abastecimiento urbano e industrial y aguas para saltos hidroeléctricos), y algo menores para demanda variable (regadío y demanda real). A ellos habría que sumar el aumento de la evaporación en los embalses.

El diseño de actuaciones hidrológicas, superficiales o subterráneas, sin considerar los resultados que se producirían con "Cambio Climático", conduciría a una sobreestimación de las demandas que podrían satisfacerse, y aun "sobredimensionamiento" de presas y embalses en la mitad Sur. Los trasvases planificados en el nonato Plan Hidrológico Nacional de 1993, serían globalmente inviables para mediados del siglo que viene por falta de excendentes para trasvasar, y los del Tajo y Guadiana para el 2020. (SOTELO NAVALPOTRO, José Antonio: "Plan Hidrológico vs Plan Hidráulico Nacional" (2001) pág: 13-16 ob. Cit. en Bibliografía)

\subsection{UNA NUEVA CULTURA DEL AGUA}

El vigente modelo neoliberal de globalización, lejos de frenar la degradación ecológica, reducir las desigualdades y garantizar a los más pobres derecho fundamentales, ha entregado la gestión del agua al mercado, convirtiendo esta gestión en una nueva oportunidad de negocio, acelerando la depreciación de los recursos hídricos y aumentando la vulnerabilidad de los más débiles.

En la actualidad se estima que 1.200 millones de personas no tienen acceso garantizado al agua potable, y de mantenerse las tendencias vigentes, el número alcanzaría los 4.000 millones en 2025. La generalizada degradación de los ecosistemas acuáticos continentales subyace como clave de este desastre humanitario. Esta crisis de insosteniblidad agrava además los problemas de hambre en el mundo, al arruinar las pesquerías (fluviales y marinas) y las formas tradicionales de producción agropecuaria vinculadas a los ciclos fluviales de inundación en las llanuras aluviales.

En síntesis, afrontamos una crisis global del agua por la convergencia de varias fallas:

- De sostenibilidad: por contaminación y detracciones abusivas en ríos, lagos y acuíferos, construcción de grandes obras hidráulicas y deforestación masiva.

- De inequidad y pobreza: que dispara la vulnerabilidad de las comunidades más pobres frente a la quiebra de los ecosistemas acuáticos.

- De gobernanza: por los problemas de corrupción y las presiones de privatización de los servicios de agua y saneamiento.

- De institucionalidad democrática global: que permita hacer del agua un espacio de colaboración entre los pueblos y no de confrontación y dominación.

Una crisis global que sin duda se agravará por efecto del cambio climático si no se adoptan adecuadas políticas de adaptación que amortigüen la vulnerabilidad de la población, particularmente de las comunidades más pobres, ante los riesgos de 
sequía y de fuertes precipitaciones que, según todas las previsiones, tenderán a aumentar, tanto en intensidad como en frecuencia.

En este contexto, más allá de impulsar cambios político-institucionales y mejoras tecnológicas, se requiere un nuevo enfoque ético, basado en principios de sostenibilidad, equidad y no-violencia. Nos encontramos, pues, ante la necesidad de promover una "Nueva Cultura del Agua" que recupere, desde la modernidad, la vieja sabiduría de las culturas ancestrales basadas en la prudencia y en el respeto a la naturaleza.

\subsection{DEL PRODUCTIVISMO A LA EFICIENCIA ECO-SOCIAL}

A pesar de la gravedad, estos impactos no suelen reflejarse en las estadísticas económicas oficiales, en la medida en que buena parte de esos alimentos se dirige a mercados locales y al autoconsumo, sin entrar en los grandes circuitos comerciales.

Se suele argumentar, por otro lado, que estos modelos de producción, vinculados a los ciclos fluviales y a técnicas artesanales de pesca, son ineficientes. No obstante, si se contabilizan los valores ambientales y sociales en juego y se asumen objetivos de sostenibilidad, distribución equitativa y acceso efectivo a los alimentos por parte de los más pobres y vulnerables, esa pretendida ineficiencia se torna en altos niveles de eficiencia eco-social.

Sin duda, es necesario cambiar el enfoque tradicional de los organismos internacionales relacionados con la alimentación en lo que se refiere a la gestión de aguas. La mitificación productivista del regadío ha llevado, por un lado, a ignorar los impactos de las políticas hidráulicas sobre las pesquerías, al tiempo que, por otro lado, se ha tendido a centrar la solución del hambre en el crecimiento del regadío. Todavía se enfatizan los diagnósticos que centran la pretendida solución en incrementar la producción de alimentos, olvidando que, a menudo, el problema es de acceso de los más pobres a los alimentos que de hecho existen. Afortunadamente, cada vez se abre más espacio a oros enfoques. Como los que desde hace años viene haciendo Vía Campesina, enfatizando la denuncia de la pobreza y la necesidad de proteger las formas de vida tradicionales que vertebran el medio rural, particularmente en los países empobrecidos y en desarrollo, frente a la agresividad del neoliberalismo imperante en el modelo de globalización vigente. O como aquellos que identifican la quiebra de los ciclos naturales que regeneran la fertilidad de la tierra, de los mares y de los ecosistemas acuáticos continentales, como clave del problema.

\subsection{EL ENFOQUE ECOSISTÉMICO}

Hace escasamente diez años, proponer que el agua fuera considerada como un activo eco-social (donde la raíz "eco" expresa al tiempo valores económicos y ecológicos), y no simplemente como un puro input productivo, era motivo de debate y controversia. Hoy, la necesidad de ese cambio conceptual nos coloca ante el reto de pasar de los tradicionales enfoques de "gestión de recurso" a nuevos enfoques de "gestión ecosistémica". Casi todo el mundo ha entendido la necesidad de pasar de la gestión maderera (gestión de recurso) a enfoques más complejos de gestión forestal (gestión ecosistémica). Un cambio parecido se hace cada vez más evidente en materia de aguas. 
De hecho, la Directiva Marco de Aguas (DMA), vigente en la Unión Europea desde finales del año 2000, promueve este nuevo enfoque, estableciendo como objetivo central recuperar y conservar el buen estado ecológico de ríos, lagos y humedales. No se trata sólo de preservar la calidad físico-química del agua, como recurso, sino de recuperar y cuidar la salud de los hábitats acuáticos y ribereños. De esta forma, más allá de los indicadores físico - químicos, emergen los indicadores biológicos. La biodiversidad pasa a ser el mejor testigo, no sólo de la calidad de las aguas, sino del buen funcionamiento de los ecosistemas.

Es necesario, por tanto, establecer nuevos criterios sociales y ambientales que permitan delimitar qué explotaciones agrarias merecen hoy ser consideradas como verdaderas actividades económicas de interés general. Consolidar el tejido rural, con sus valores sociales, culturales y paisajísticos, o favorecer la consecución de determinados objetivos ambientales, serían, sin duda, argumentos de interés general en la sociedad actual. En este sentido, resulta razonable argumentar el interés general de proteger la explotación familiar agraria, en particular en el regadío. Pero sería necesario unir a ese argumento social, la exigencia de buenas prácticas agroambientales.

La UE empieza a promover, aunque tímidamente, políticas agroambientales y criterios de eco-condicionalidad en materia de subvenciones. Desde estos enfoques debe revalorizarse el secano, como práctica agro-ambiental, particularmente en el área mediterránea, donde se han rebasado los límites de sostenibilidad de nuestros ríos y acuíferos. En este caso, en las vigentes condiciones de cambio climático, más allá de revisar de forma realista y prudente el actual Plan Nacional de Regadío, sería necesario elaborar un Plan Nacional de Secano que establezca políticas concretas en este campo.

En todo caso, y aun aceptando la necesidad de apoyar determinadas actividades agrarias en el regadío, sería vital reflexionar sobre cómo realizar tales ayudas, de forma que se induzcan buenas prácticas y actitudes responsables. En concreto, sería preferible subvenciones directamente a las correspondientes actividades productivas, en lugar de ofrecer agua subvencionada, como suele hacerse. De esta manera, con el mismo coste para la hacienda pública, se induciría un uso más eficiente y responsable del agua.

Hoy, más allá del reconocimiento formal del dominio público sobre las aguas y los ecosistemas hídricos, nos encontramos ante la necesidad de reflexionar sobre los retos que imponen, tanto el nuevo paradigma de sostenibilidad, como la obligación de garantizar el acceso al agua potable y a servicios básicos de saneamiento como un derecho humano.

Asumir en materia de gestión de aguas los principios de equidad inter e intrageneracional, refuerza la necesidad de replantear el dominio y la gestión pública o comunitaria sobre los ecosistemas hídricos y los acuíferos, desde nuevos enfoques que garanticen la prioridad de garantiza de forma sostenible sus funciones de vida, así como los derechos humanos, incluidos los de las generaciones futuras. (ARROJO AGUDO, Pedro: "Crisis global del agua: valores y derechos en juego" Cuadernos CJ nº 168, págs. Barcelona, 2010 págs:3-32) 


\section{CONCLUSIONES}

A tenor de la publicación La huella hídrica española en el contexto del medio ambiental dirigida por el Profesor José Antonio Sotelo Navalpotro, llegados a este punto podemos afirmar al tratar de la Huella Hídrica española, en el contexto del Desarrollo y del Medio Ambiente, que transmisor de un mensaje espiritual del pasado, el agua se integra en el patrimonio monumental de los pueblos, permaneciendo en la vida presente como testimonio vivo de sus perennes tradiciones. Nuestro admirado y añorado profesor Ángel Ramos, al respecto nos enseñó que la degradación o destrucción de estas obras, en aras de un hipotético progreso -entendido por lo general como económico, eso sí, "sostenibles"-, supone un desastre y un auténtico drama valorable a escala mundial, mas con notables consecuencias en España. Todo ello sin olvidarnos de que el susodicho patrimonio se integra, como una aportación más del hombre en la naturaleza; en este sentido, la historia lejana y reciente nos muestra con notable claridad las consecuencias de errores ecológicos en la utilización de suelos, bosques, recursos hidrológicos, localización de ciudades y factorías, en el exterminio de recursos marinos, desaparición de especies, Estos y otros atentados contra el patrimonio ecológico son, también, malos negocios evitables en la medida en que se conozcan sus relaciones causa-efecto, en y sobre nuestro patrimonio (natural, artístico y cultural). A este respecto, recordar que en España se ha insistido mucho en los últimos años en la necesidad de la conservación, olvidándonos en no pocos que ésta no supone, necesariamente, caer en actitudes pasivas o negativas, sino que por el contrario requiere de unos conocimientos y unas operaciones inteligentes, adecuados a cada circunstancia.

Debemos considerar que la notabilísima degradación medioambiental de los medios rurales y urbanos, de los monumentos y de todos los vestigios del pasado, son fruto del denominado "progreso tecnológico", a la par que de la crisis de la cultura humanística -subyugada por los encantos del neoliberalismo-; esta tendencia, únicamente, podrá detenerse si se logra un desarrollo más ecuánime, más racional, siempre mediante la puesta en práctica de una educación más cívica, estética y medio ambiental. La cultura es el hilo conductor que une el pasado, el presente y el futuro en los espacio culturales. Sin embargo, respecto del medio ambiente, como señaló el profesor González Bernáldez, desde la década de los sesenta del pasado siglo asistimos a un proceso de divulgación en los sectores más amplios del análisis de sistemas tal como se practica en Ecología y en Geografía. Por el contrario, las realizaciones serias -en el proceso que podríamos llamar "racionalización del uso de la biosfera" son escasas. Con frecuencia el público tienen una idea deformada -manipulada- de los problemas de la gestión ecológica de los recursos ambientales. Es muy notable la importancia política de la copia y mimetismo de una serie de "clichés", provenientes del pensamiento único hoy dominante, sin que se advierta ningún esfuerzo de adaptación de los problemas a las circunstancias locales, a la vida real. Frente al esnobismo, tremendismo y frivolidad tan difundidos en este terreno, la Ecología tuvo unos orígenes muy serios. E. Haeckel definidor del término "ecología", Moebius, que subraya la importancia y entidad de la "biocenosis" como conjunto donde se gestan determinados recursos, y más recientemente Tansley, creador del concepto de "eco- 
sistema", se movían en un terreno científico, pero al mismo tiempo, preveían las aplicaciones prácticas de esas ideas. Si los recursos naturales, se crean y se mantienen en funcionamiento será interesante para una gestión racional del patrimonio natural. La escasez de agua no se soluciona ni sobreexplotando los recursos subterráneos, ni aumentando los recursos regulados a través de embalse, ni importando agua mediante trasvases, pues ninguno de ellos aportan soluciones definitivas, ni hacen crecer la demanda de forma descontrolada al aumentar la oferta. Todo ello sin olvidarnos de la extrema importancia que debemos dar al agua, primando la gestión racional y sostenible del recurso, la disminución de los consumos, mejorar la eficiencia de los usos, ..., cuestiones de vital importancia para España y Portugal.

\section{BIBLIOGRAFÍA}

AEMA: Los recursos de Agua en Europa: como hacer frente a la escasez del agua y la sequía. Edita Ministerio de Medio Ambiente, Medio rural y Marino. Madrid, 2010. Págs. 55.

AEMA, 2010. El medio ambiente en Europa: Estado y perspectivas 2010 - Sintesis. Agencia Europea de Medio Ambiente, Copenhague. Pags. 222

A.A.V.V.: Documento final de las Semanas temáticas TRIBUNA DEL AGUA. ExpoZaragoza 2008, págs: 496

A.A.V.V.: Semana de estudio de los recursos del Mar Conferencias y circulares. Cartagena, Nov. 1983, págs: 106

ALCOLEA MORATILLA, M. ÁNGEL; GARCÍA ALVARADO, J.M. "El agua en la Comunidad de Madrid" en Rev. OBSERVATORIO MEDIO AMBIENTAL, núm. 9, Madrid, 2006, págs: 63-96

ÁLVAREZ COBELAS, MIGUEL: "Ecología acuática de los humedales de la Comunidad de Madrid. Rev. OBSERVATORIO MEDIO AMBIENTAL, núm.6, Madrid, 2003, págs: 99-120

ARROJO AGUADO, PEDRO: "Crisis global del agua: valores y derechos en juego" Cuadernos CJ, núm 168, Barcelona, 2010, págs: 3-32

ASOCIACIÓN ESPAÑOLA DE EDUCACIÓN AMBIENTAL: "IV Congreso Internacional de Educación Ambiental. El agua en la Educación Ambiental en la década del Desarrollo Sostenible. Madrid, 10-12 de septiembre de 2008.

AZQUETA, DIEGO, LANDA, LUCÍA y TIRADO, SERGIO: "La política ambiental en España: un nuevo rumbo, viejos problemas" en Rev. ECONOMISTAS n 104. Extra año XXIII, España, 2004 págs: 262-267

BANCO DE BILBAO, EL CAMPO. Boletín de información agraria: Oct-Dic 1984, n 96 , págs: 123

BIACONI, MARIO: “Ambiente y ecosostenibilidad” págs 157-165 en MARÍN; Juan, DACLO, Corrado María Per un zinnovato sapere delle sienze ambientali. Scienza e etica per l'ambiente nel terzo milenio. Edit. Franco Angeli. Milano, 2005.

CALDERÓN BALANZATEGUI, ENRIQUE: "La Estrategia Española del Desarrollo Sostenible y las políticas de gestión de recursos hidráulicos en el litoral mediterráneo español" Rev. OBSERVATORIO MEDIO AMBIENTAL, núm. 6, Madrid, 2003, págs: $121-140$ 
COMISIÓN DE LAS COMUNIDADES EUROPEAS: Hacia un Desarrollo Sostenible. V Programa de acción en materia de Medio Ambiente y Desarrollo Sostenible, Bruselas, 20 de mayo de 1992, pág: 107

COMISIÓN DE LAS COMUNIDADES EUROPEAS: Sexto Programa de Acción de la Comunidad Europea en materia de Medio Ambiente. Medio Ambiente el futuro está en nuestras manos. Bruselas, 24 de enero de 2001.

CONDESSO, FERNANDO: Desarrollo y cohesión en la Península Ibérica. El problema de la ordenación territorial. Erasmus Ediciones, $1^{\text {a }}$ edición, Barcelona, mayo 2010, págs: 252

DE ESTEBAN ALONSO, ALFONSO: "Agua y ruido: retos ambientales para el siglo XXI" en LÓPEZ LÓPEZ, Alejandro: Medio Ambiente y desarrollo Sostenible en los países mediterráneos de la Unión Europea, págs: 239-268. Edita Fundación Biodiversidad - Instituto Universitario de Ciencias Ambientales (UCM) Madrid, 2005

DE LA ROSA, MARÍA DEL CARMEN, MOSSO, MARÍA ÁNGELES: "Historia de las aguas mineromedicinales en España" en Rev. Observatorio Medioambiental num. 7, Madrid, 2004, págs: 117-137

DIARIO OFICIAL DE LA COMUNIDADES EUROPEAS: Directiva del Consejo de 8 de diciembre de 1975 relativa a la calidad de las aguas de baño (76/160 CEE)

DIRECCIÓN GENERAL DE CALIDAD Y EVALUACIÓN AMBIENTAL: Uso sostenible del agua en Europa, Ministerio de Medio Ambiente de España. Madrid, 2006.

DOCUMENTOS EUROPEOS: La Comunidad Europea y los problemas del agua. Mayo, 1989 8/89

EGEA, ALEJANDRO, POVEDA, ÁNGEL, GIL OLCINA, ANTONIO, MELGAREJO, JOAQUÍN: DOSSIER EL AGUA. Veinte siglos de un País sediento Rev. La Aventura de la Historia ${ }^{\circ} 105$, Julio 2007, págs: 70-93

EUROPA, JUAN: Andalucía: Consejería de Presidencia, no 131 (2010) "Calidad de las aguas europeas" págs: $22-25$

FRERS, CRISTIAN: "El uso de plantas acuáticas para el tratamiento de aguas residuales" en Rev. OBSERVATORIO MEDIO AMBIENTAL, núm. 11, Madrid, 2008, pags: 301-305

FURÓN, RAYMOND: El agua en el mundo Alianza Editorial. Libro de bolsillo ${ }^{\circ}$ 47, Madrid, 1967.

GARCÍA GARCÍA, JESÚS: Guia legal del Medio Ambiente en España" Amarú ediciones, Salamanca, 1993, 256 págs.

GÓMEZ, RICARDO, DEL PALACIO, MAMPASO y SÁNCHEZ SEGURA, TERESA: "Un ejemplo de abastecimiento y saneamientos a ciudades. La Confederación Hidrográfica del Tajo" en Rev. OBSERVATORIO MEDIO AMBIENTAL, núm. 8, Madrid, 2005, págs: 127-151.

GONZÁLEZ VALLVÉ, JOSÉ LUIS y BENEDICTO SOLSONA, MIGUEL ÁNGEL: La mayor operación de solidaridad de la historia. Crónica de la politica regional de la Unión Europea en España. Oficina de Publicaciones de la Comisión Europea. Luxemburgo, 2006, 184 pp. 
HERALDO DE ARAGÓN: El Tribunal Constitucional también avala la parte hídrica del texto catalán" Sábado 11 de septiembre de 2010, pág:3

HERALDO DE ARAGÓN: Monográfico especial "50 aniversario del Canal de Bardenas 1959-2009" miércoles 8 de abril de 2009, págs: 1-8

INSTITUTO GEOLÓGICO Y MINERO: Las aguas subterráneas. Un recurso natural del subsuelo Con el patrocinio de la Fundación Marcelino Botín y el Ministerio de Educación, Cultura y Deporte. Madrid, 2001. págs: 79

JIMÉNEZ HERRERO, LUIS: "Nueva economía para un Desarrollo Sostenible" (págs: 107-132) en LÓPEZ LÓPEZ, Alejandro y BORRELL MERLIN, Maria Dolores: Medio Ambiente y Desarrollo Sostenible en los Países Mediterráneos de la Unión Europea. Editan Fundación Biodiversidad e Instituto de Ciencias Ambientales (UCM), Madrid, 2005, págs: 393

JUNQUERA RUBIO, CARLOS: “ La propiedad del aguas destinada a regadío en la ribera del Órbigo (Jaén): de la repoblación a la actualidad" en Rev. OBSERVATORIO MEDIO AMBIENTAL, núm. 9, Madrid, 2006, págs: 125-154

LA ROCA, FRANCESC; FERRER, GRACIELA: "La economía en el desarrollo de la Directiva Marco del Agua", en Rev. OBSERVATORIO MEDIO AMBIENTAL, núm. 10, Madrid, 2007, págs: 179-198

LÓPEZ LÓPEZ, ALEJANDRO: "Turismo y Desarrollo Sostenible" en Rev. SISTEMA., núms. 162-163. Madrid, junio 2001, págs:189-202

LÓPEZ LÓPEZ, ALEJANDRO "E1 medio ambiente y las nuevas tendencias turísticas: referencia a la región de Extremadura" en Rev. Observatorio Medio Ambiental no 4,Madrid, 2001, págs: 205-251.

LÓPEZ LÓPEZ, ALEJANDRO y BORRELL MERLIN, MARIA DOLORES: Medio Ambiente y Desarrollo Sostenible en los Países Mediterráneos de la Unión Europea. Editan Fundación Biodiversidad e Instituto de Ciencias Ambientales (UCM), Madrid, 2005, págs: 393

LÓPEZ VERA, FERNANDO: Contaminación de la Aguas Subterráneas Centro de Publicaciones, Ministerio de Obras Públicas y Urbanismo, Madrid, 1990, 78 págs. MAESTRE, JUAN; ROJO, TERESA: "División de la opinión pública española sobre las estrategias sostenibles del agua. Metodología EASW para la acción consensuada, Rev Observatorio Medio Ambiental, núm. 5, Madrid, 2002, págs: 193-216

MARTÍN CABO, SERGIO: "La gestión del agua" en Rev. OBSERVATORIO MEDIO AMBIENTAL, núm. 11, Madrid, 2008, págs: 279-299

MARTÍN MESA, ANTONIO y A.A.V.V.: "Las cuentas del agua en Andalucía" en Rev. OBSERVATORIO MEDIO AMBIENTAL, núm. 10, Madrid 2007, págs: 213-242

MATARÁN RUIZ, ALBERTO; PÉREZ CAMPAÑA, R.; NAVARROCANO, S. y SÁNCHEZ HITA, C: "Las Ciencias Ambientales y la nueva cultura del agua: resumen y principales conclusiones del I Congreso Andaluz de Desarrollo Sostenible (AMBIENTALIA): El Agua" en Rev. OBSERVATORIO MEDIO AMBIENTAL, núm.10, Madrid, 2007, págs: 365-377

MOHAMMADIAN, MANSOUR: "La Bioeconomía, Sostenibilidad y Globalización" en LÓPEZ LÓPEZ, Alejandro y BORRELL MERLIN, Maria Dolores: Medio Ambiente y Desarrollo Sostenible en los Países Mediterráneos 
de la Unión Europea. Editan Fundación Biodiversidad e Instituto de Ciencias Ambientales (UCM), Madrid, 2005, págs: 393

MOHAMMADIAN, MANSOUR: La Bioeconomía: Economía del Tercer camino. Entre la antigua economía y la nueva economía global. Ed. Personal, Madrid, 2008, págs: 254

MORA ALISEDA, JULIÁN: Planificación estratégica y gestión compartida de los recursos hídricos hispano-lusos. Proyecto del Plan Nacional $\mathrm{I}+\mathrm{D}$, convocatoria de 2010 de Investigación Fundamental no orientada.

MORALES GIL, ALFREDO; RICO AMORÓS, ANTONIO MANUEL; HERNÁNDEZ HERNÁNDEZ, MARÍA: "El trasvase Tajo-Segura" en Rev. OBSERVATORIO MEDIO AMBIENTAL, núm. 8, Madrid, 2005, págs: 73-110 NATIONAL GEOGRAPHIC, España: Agua, un mundo sediento, Abril 2010, 100 págs. OSE (Observatorio de la Sostenibilidad en España): Sostenibilidad en ESPAÑA. 2010 Ediciones Mundi-Prensa. Madrid, 2010, Págs. 494. Ver sobre todo el capítulo 12 Agua, Págs. 249-270.

PAVILLON BLEU, d'Europe 1990: DAl Grafik/RAPO/COPENHAGEN, 1990, 24 págs. PÉREZ GONZÁLEZ, MARÍA INMACULADA y GARCÍA RODRÍGUEZ, PILAR: "Aplicaciones de la teledetección en hidrología" en la Rev. OBSERVATORIO MEDIO AMBIENTAL, núm. 9, Madrid, 2006, págs: 171-186

PÉREZ PÈREZ, FÉLIX y PÉREZ GUTIÉRREZ, JOSÉ FÉLIX: “Agua y Medio Ambiente" en Rev. OBSERVATORIO MEDIO AMBIENTAL, núm. 9, Madrid, 206, págs: $9-25$

RÍO92: Edita Ministerio de Obras Públicas y Transportes (España), Madrid, 1993 RETAMA (Revista Técnica de Medio Ambiente) Sep-Oct. 1987.

SANCHEZ MARTÍNEZ, FRANCISCO JAVIER (Coordinador): Programa de Eduación Ambiental y Voluntariado en ríos, actuaciones 2009 Edita Dirección General del Agua, Ministerio de Medio Ambiente, Medio rural y Marino, Madrid, 2010. Págs. 89

SANZ DONAIRE, JUAN JOSÉ: "Indicadores pluviométricos de sequía para España (1865-2004)" en Rev. OBSERVATORIO MEDIO AMBIENTAL, núm. 9, Madrid, 2006, págs: 187-213

SOTELO NAVALPOTRO, JOSÉ ANTONIO: "Plan hidrológico vs Plan Hidráulico Nacional" Rev. OBSERVATORIO MEDIO AMBIENTAL, núm. 4, Madrid, 2001, págs: $13-16$

SOTELO NAVALPOTRO, JOSÉ ANTONIO: "Desarrollo y Medio Ambiente en el municipio de Madrid: Abastecimiento y saneamiento urbano" en Rev. OBSERVATORIO MEDIO AMBIENTAL, núm. 9 Madrid, 2006, págs: 215-264

SOTELO NAVALPOTRO, JOSÉ ANTONIO (Elaboración): La huella hídrica espanola en el contexto del cambio ambiental. Fundación Mapfre, Madrid, diciembre 2010. Págs. 216.

UNESCO: La Naturaleza y sus recursos. Boletín del Programa Hidrológico Internacional y la Conferencia de Naciones Unidas sobre el Agua (Mar del Plata, Argentina, 14 al 25 de marzo de 1977) págs: $24-28$ Vol. XIII, $n^{\circ}$ 2, abril-junio 1977, págs: 43 
UNESCO "Cuadernos de Educación Ambiental: Mares y Océanos", Todos, 10 junio 1994.

VALENZUELA MONTES, LUIS MIGUEL ; MATARÁN RUIZ, ALBERTO: "Criterios e indicadores para la formulación de un Observatorio de Desarrollo Territorial de las relaciones entre agua, agricultura y paisaje" en Rev. OBSERVATORIO MEDIO AMBIENTAL, núm. 10, Madrid, 2007, págs: 243-263

VANGUARDIA, Dossier: Agua el desafio del siglo XXI, núm. 21 octubre-diciembre 2006, Barcelona, págs. 130. 